\title{
ORIGINAL RESEARCH \\ Infantile Hemangiomas Involving the Neuraxis: Clinical and Imaging Findings
}

V. Viswanathan

E.R. Smith

J.B. Mulliken

S.J. Fishman

H.P.W. Kozakewich

P.E. Burrows

D.B. Orbach
BACKGROUND AND PURPOSE: The neuroradiology and neurosurgery literature is replete with references to "hemangioma" involving the central nervous system (CNS). However, the number of cases of true infantile hemangiomas in the CNS reported to date is 15 . Our purpose was to delineate the definition of infantile hemangiomas, determine their prevalence in the neuraxis, and describe their imaging characteristics and associations in this location.

MATERIALS AND METHODS: We reviewed our Vascular Anomalies Center data base from 1999 through May 2008 to assess the prevalence of intracranial or intraspinal involvement within the total cohort of infantile hemangiomas. Fifteen patients were identified with infantile hemangiomas that involved the neuraxis. Two board-certified neuroradiologists reviewed the available imaging of these 15 patients, and a board-certified pathologist reviewed the available histopathology. Clinical records of all 15 patients were reviewed to identify the type of treatment and the treatment response.

RESULTS: Of the 1454 patients listed with infantile hemangioma, $15(\sim 1.0 \%)$ had involvement of the CNS. Eight patients had intracranial infantile hemangioma, 6 had intraspinal hemangioma, and 1 had both. In most instances, there was continuous extension into the neuraxis from an extracranial or extraspinal lesion. There were no cases of a CNS hemangioma without an accompanying extra-CNS tumor. Two patients had findings consistent with posterior fossa anomalies, cervicofacial hemangioma, arterial anomalies, cardiac defects, ocular abnormalities, and associated sternal or ventral defect. Of note, there were no brain or spinal parenchymal signal-intensity abnormalities, and there was no evidence of parenchymal invasion.

CONCLUSIONS: CNS involvement by infantile hemangiomas is an unusual occurrence, which, when recognized, can help optimize patient management.
$\mathbf{T}$ he classification of vascular anomalies proposed by Mulliken and Glowacki in $1982^{1}$ was adopted by the International Society for the Study of Vascular Anomalies (ISSVA) in 1996. ${ }^{2}$ There are 2 major categories in this system based on clinical and cellular behavior: vascular tumors (which arise by endothelial hyperplasia, such as infantile hemangioma) and vascular malformations (which arise by dysmorphogenesis and exhibit normal endothelial turnover, unless perturbed).

Cutaneous hemangioma, the most common tumor of infancy, presents in the first few weeks of life, with the proliferative phase reaching a plateau at approximately 9-10 months of age, followed by a slow involuting phase over the next 5-7 years. Deep cutaneous or visceral lesions may not become apparent until several months of age. Most uncomplicated infantile hemangiomas do not require treatment. Endangering, distorting, or destructive hemangiomas are treated pharmacologically (with corticosteroid, interferon, or vincristine). There are only scattered reports of true infantile hemangiomas involving the central nervous system (CNS). ${ }^{3-12}$

Approximately $60 \%$ of infantile hemangiomas occur in the craniofacial region. ${ }^{13}$ The lesions are described as superficial, deep, or of combined depth. Focal lesions are distinguished

Received September 22, 2008; accepted after revision December 2

From the Neuroradiology Division (V.V., D.B.O.), Brigham and Women's Hospital, Boston, Mass; Neurosurgery Division (E.R.S.), Vascular Anomalies Center (J.B.M., S.J.F., H.P.W.K, D.B.O.) and Neurointerventional Radiology (D.B.O.), Children's Hospital Boston, Boston, Mass; and Vascular Malformations Center (P.E.B.), Roosevelt Hospital, New York, NY.

Please address correspondence to Darren Orbach, MD, PhD, Neurointerventional Radiology, Brigham and Women's Hospital, 75 Francis St, PBB-356, Boston, MA 02115; e-mail: dorbach@partners.org

$\equiv$ indicates article with supplemental on-line table

DOI 10.3174/ajnr.A1478 from larger regional lesions (ie, extensive plaquelike hemangiomas that occupy a facial region). The classic bright red elevated noncompressible plaque of proliferative phase focal infantile hemangioma as seen on physical examination is demonstrated in Fig 1A. A "reticular" variant, consisting of a large flat pink stain with a macular network-like pattern, has also been described and is illustrated in Fig $1 B .{ }^{11}$

Infantile hemangiomas have a characteristic appearance on cross-sectional imaging and angiography, depending on the stage of their natural history. During the proliferative phase, CT and MR imaging demonstrate a well-circumscribed lobular homogeneous soft-tissue mass, which shows intense and persistent enhancement. Proliferating hemangiomas are isointense or hypointense to muscle on T1-weighted images and moderately hyperintense with associated flow voids on T2-weighted images. ${ }^{14}$ Internal signal-intensity heterogeneity, occasionally seen, may reflect thrombus or prior hemorrhage. ${ }^{15,16}$ Doppler sonography demonstrates numerous intralesional and perilesional vessels with a high peak arterial Doppler shift. ${ }^{17,18}$ Angiography, though rarely performed, demonstrates enlarged arterial feeders and intense contrast staining, with pooling of contrast within tumoral vascular spaces. ${ }^{19}$ Early draining veins may also be present, with occasional unambiguous arteriovenous shunting. During the involuting phase, hemangiomas enhance to a lesser degree, are associated with fewer radiographic signs of fast-flow vascularity, and appear heterogeneous. Fibrofatty tissue characterizes the final involuted phase. Intracranial and intraspinal infantile hemangiomas have been shown to exhibit the same cross-sectional imaging characteristics as cutaneous hemangiomas.

By systematically reviewing our entire cohort of infantile hemangiomas, we hope to demonstrate that these lesions have 

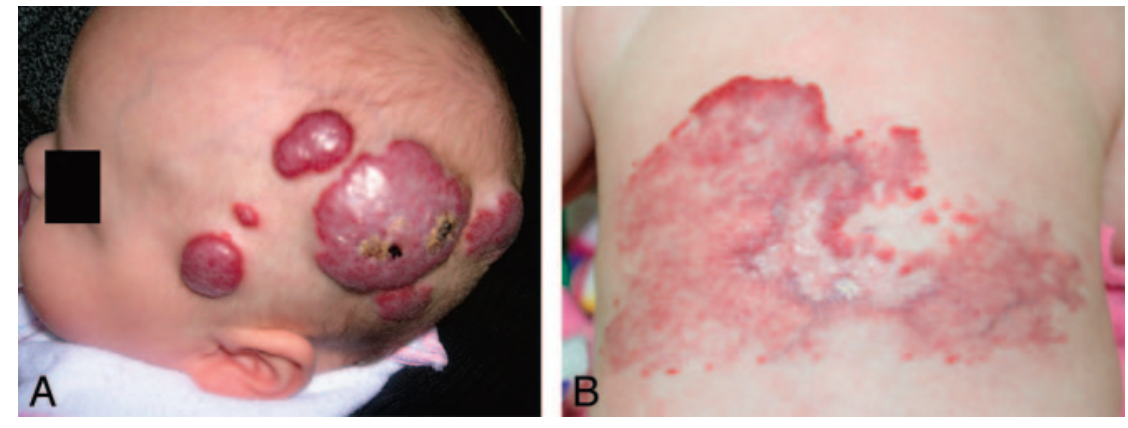

Fig 1. Gross appearance of cutaneous lesions overlying infantile hemangiomas involving the neuraxis (patient 12). A, A 4-month-old female infant presented with multiple bleeding scalp hemangiomas and anemia (patient 3). B, Another 4-month-old female infant presented with a large flat pink stain over the lower back, with a macular network-like structure consistent with the reticular pattern of spinal hemangioma. This appearance has been associated with underlying ventral-caudal structural anomalies.

a unique natural history, which should not be confused with other vascular lesions; a unique response to appropriate therapy; and a specific pattern of CNS involvement.

\section{Materials and Methods}

We reviewed all of the records of our Vascular Anomalies Center data base from 1999 through May 2008, most of which were referrals from outside sources, by using the search terms "hemangioma," "head and neck," "intracranial," "periorbital," "skull base," "intraspinal," "spinal," "coccygeal," "PHACE," and "PHACES." We identified a total of 1454 patients with a diagnosis of infantile hemangioma based on clinical and imaging characteristics. In this cohort, there were 15 patients in whom infantile hemangioma involved the intracranial or intraspinal compartments. Clinical records and imaging studies for all 15 patients were reviewed. Four patients were imaged at Children's Hospital Boston. Eleven patients were imaged at referring institutions, from whom we obtained digital copies ( 9 patients) or hard copies ( 2 patients) of cross-sectional imaging.

The 4 patients imaged in our institution were scanned on a $1.5 \mathrm{~T}$ MR imaging system (LX Twinspeed; GE Healthcare, Milwaukee, Wis). Standard sequences at our institution include pre- and postcontrast T1, T2, fluid-attenuated inversion recovery, diffusion-weighted, and gradient-echo imaging. MR imaging alone was available for 12 of the 15 patients $(80 \%)$, CT imaging alone was available for 2 of the 15 patients (13\%), and both CT and MR imaging were available for 1 patient $(7 \%)$. One patient had sonographic imaging of the lumbosacral spine to rule out tethered cord. Both initial and follow-up imaging was available for 9 of 15 patients (60\%), whereas only initial imaging was available for 6 patients (40\%). All imaging was reviewed independently by 2 board-certified neuroradiologists. The lesions were assessed for T1- and T2-weighted imaging characteristics, morphology, extent of contrast enhancement, the presence of flow voids, the location of infantile hemangioma outside the CNS, location within the CNS, continuity between the extra-CNS and CNS components, and important associated CNS findings. Histopathologic confirmation was available for 2 patients and was reviewed by our pathologist at Children's Hospital Boston. In all cases, clinical history and imaging characteristics were consistent with infantile hemangioma. All 15 patients' clinical records were reviewed to identify the treatment each patient received and the response.

\section{Results}

Clinical and imaging characteristics of the 15 patients are summarized in the on-line Table.

\section{Clinical Features}

Eleven of the 15 patients (73\%) were female and 4 (27\%) were male. Eight patients had intracranial hemangioma, 6 had intraspinal hemangiomas, and 1 had tumor in both regions. All the lesions of the head and neck were focal, either solitary or multiple, with no single lesion occupying an entire facial region. Two of the patients with intraspinal involvement had a cutaneous reticular hemangioma on the back. Most patients presented with cutaneous tumors within the first few weeks of life (Fig 1). Six patients had nascent superficial cutaneous lesions at birth. One patient had a deep scalp lesion, which did not appear until 4 months of age. One patient had hemangiomatosis complicated by congestive heart failure. Of the 8 patients with intracranial hemangioma, 1 exhibited irritability and lethargy without focal neurologic deficit, whereas the remaining 7 patients had no neurologic signs or symptoms. Of the 6 patients with intraspinal hemangioma, 2 presented with neurologic deficits at birth: 1 with decreased deep tendon reflexes, rockerbottom soles, and decreased lower extremity motor function; and the other with abnormal bladder function. The patient with both intracranial and intraspinal hemangioma presented with a right-sided facial skin lesion, but had no accompanying neurologic deficits.

\section{Imaging Features}

Several important features of infantile hemangioma were noted on cross-sectional imaging. In all cases, the hemangiomatous component within the neuraxis had the same imaging features as the extracranial/extraspinal appearance (ie, isointensity or hypointensity on T1-weighted images with avid uniform enhancement and hyperintensity on T2-weighted images). Typical MR imaging findings are illustrated in Fig 2. Thirteen lesions had prominent intralesional flow voids, characteristic of the proliferative phase (Fig 3 ). In the 3 patients who underwent CT imaging, the CNS hemangiomas were isoattenuated to hyperattenuated, with a lobulated morphology and intense enhancement. Typical CT findings are demonstrated in Fig 4.

Intracranial infantile hemangioma insinuated within the basilar cisterns and intraspinal lesions involved the extradural space, without parenchymal involvement. All 9 patients with intracranial hemangiomas had tumor in the basal cisterns. These included the cerebellopontine angle cistern (Figs 2 and 5 ), the prepontine cistern, the perimesencephalic cistern, and 

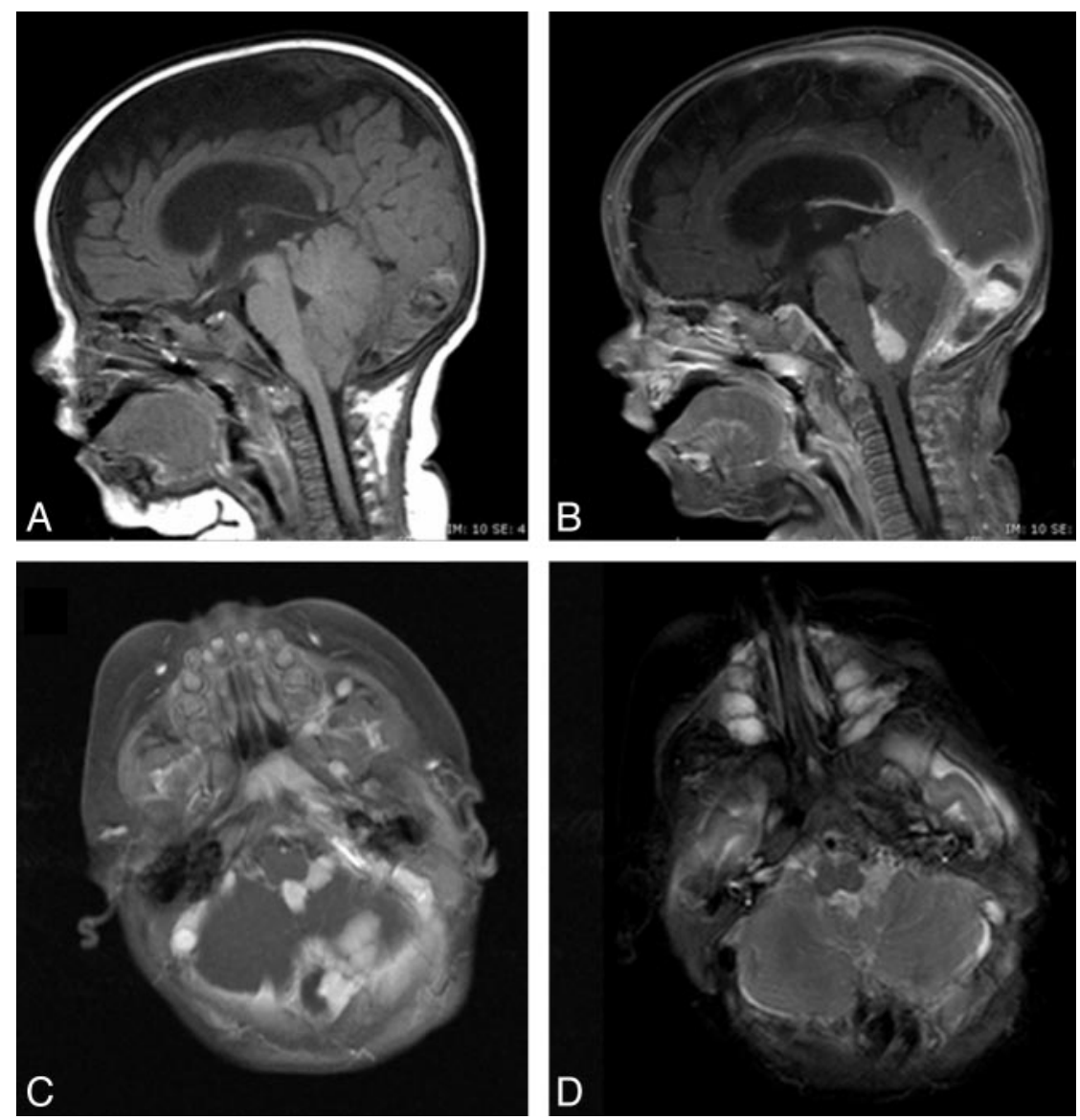

Fig 2. Typical MR imaging findings of neuraxis hemangioma (patient 3). Sagittal precontrast $(A)$ and postcontrast $(B)$, and axial postcontrast $T 1$ - $(C)$ and $T 2$-weighted $(D)$ images demonstrate a well-circumscribed lesion in the fourth ventricle with extension into the left foramen of Luschka. The lesion is isointense on T1-weighted imaging, hyperintense on T2-weighted imaging, and enhances avidly and uniformly. Note the lack of normal enhancement within the torcular herophili, consistent with thrombosis.

the quadrigeminal plate cistern. One patient had an extensive hemangiomatous coating of the prepontine, perimesencephalic, and suprasellar cisterns and of the sella turcica. Four patients had enhancing hemangioma involving the cavernous sinus (Fig 4), and 3 patients had enhancing hemangioma within the fourth ventricle (Figs 2 and 5). In all 7 patients with intraspinal hemangioma, the tumor was extradural (Fig 3). None of the neuraxial hemangiomas invaded the brain or spinal cord parenchyma or exerted significant mass effect.

On cross-sectional imaging, continuity between extra-CNS and CNS components of infantile hemangioma could be established in most patients. Of the 9 patients with intracranial hemangioma, continuity was seen in 6 . In the other 3 patients, though continuity could not be demonstrated, the cutaneous tumor overlay the intracranial tumor. The bridging anatomic structures included the superior orbital fissure $(n=3)$ (Fig 4), the inferior orbital fissure $(n=2)$, the foramen rotundum $(n=1)$, and the hypoglossal canal $(n=1)$. Of the 7 patients with intraspinal infantile hemangiomas, 4 had continuity between the extraspinal and extradural intraspinal tumors. In the remaining 3 patients, continuity could not be demonstrated, but similar to the intracranial cases, the cutaneous tumor overlay the intraspinal tumor.

Clinically important associations were seen in several of our patients with infantile hemangiomas. Hydrocephalus was noted in 3 patients. Thrombosis of the right transverse sinus occurred in another patient (Fig 2). Hypoplasia of the cerebellar hemisphere ipsilateral to the hemangioma was documented in 2 patients and Dandy-Walker cyst, in another. Tracheal impingement was seen in 1 patient, and an intratracheal lesion was noted in another. One patient had diffuse gastrointestinal hemangiomatosis, and 2 patients had multiple hepatic hemangiomas. Three patients had a constellation of findings consistent with posterior fossa anomalies, cervicofacial hemangioma, arterial anomalies, cardiac defects, eye abnormalities, and sternal or ventral defects (PHACES) association, including 1 with a moyamoya-like proliferation of vessels at the left internal carotid artery (ICA) terminus, with extension of hemangioma into the left cavernous sinus (Fig 4). Subependymal cysts were found in 1 patient. Two of the 7 spinal patients had a tethered cord. One of these patients had an associated lipoma, and the other had an associated intramedullary angiolipofibroma. Both of these latter lesions were distinct from the intraspinal hemangioma.

\section{Histopathologic Features}

Two patients with intraspinal hemangiomas involving the lumbosacral spine had histopathologic confirmation. In patient 10 in the on-line Table, the excised subcuticular extradural tissue consisted of a proliferative-phase hemangioma admixed with adipose tissue. The hemangioma had a lobular, 

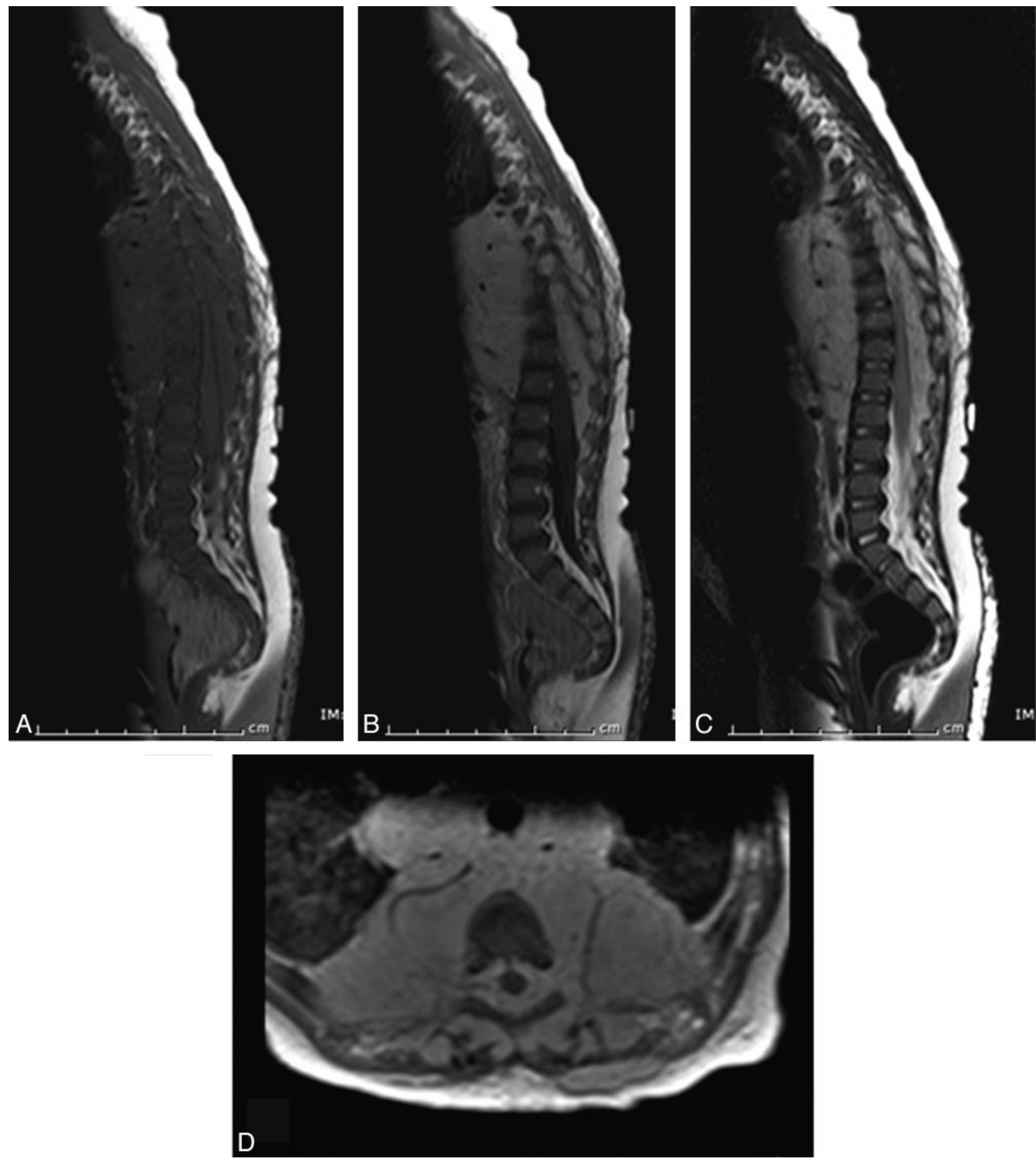

Fig 3. Infantile spinal hemangioma (patient 14). Sagittal T1 precontrast (A), postcontrast (B), and T2-weighted (C) images and an axial postcontrast T1-weighted image (D) demonstrate avidly enhancing hemangioma within the extradural compartment of the lower thoracic and upper lumbar spine. Note the prominent flow voids indicative of the proliferative phase of infantile hemangioma.

solid, and infiltrative pattern with small capillaries, plump endothelium, and a thin basement membrane. Endothelial cells were immunopositive for GLUT1, a reliable marker for infantile hemangioma. ${ }^{20}$ This patient had imaging evidence of regression of the lesion after treatment. In patient 13 in the online Table, the excised skin, subcutis, and tract to the tethered cord had lobules of hemangioma in its early involutive phase. The capillaries had large lumens, flattened endothelium, and a thick basement membrane. Associated arteries and veins were slightly prominent, and the subcutis also had foci of embryonic fat and cartilage.

\section{Treatment}

Ten patients were treated with oral corticosteroid. In 2 patients, systemic corticosteroid therapy was supplemented with interferon- $\alpha$, and 1 of these patients subsequently developed spastic diplegia, a known potential adverse effect. In another patient with intracranial hemangioma, interferon- $\alpha$, admin- 

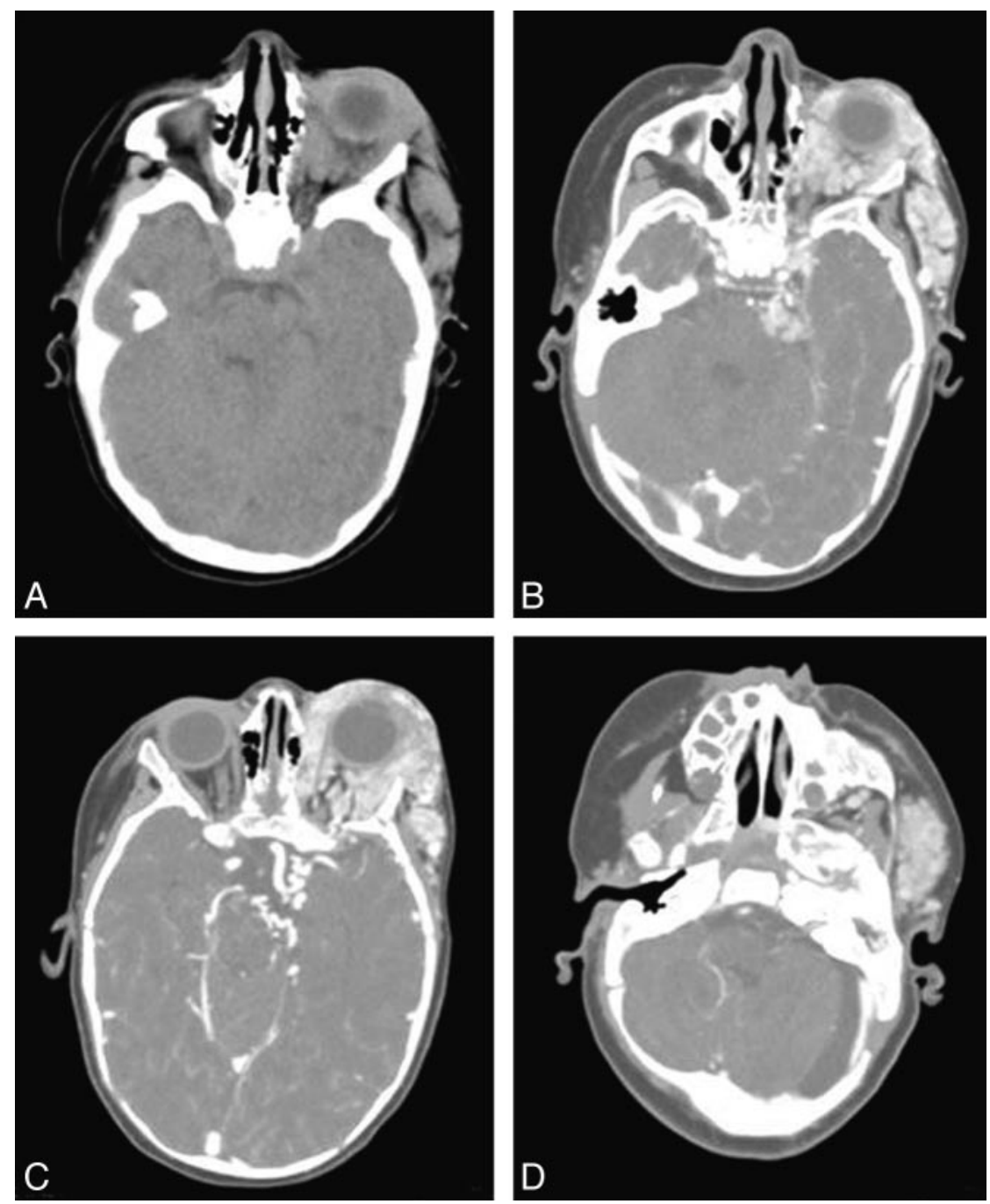

Fig 4. Typical $C T$ findings of neuraxis hemangioma (patient 2). Precontrast $(A)$ and postcontrast $(B-D)$ images demonstrate an isoattenuated lobulated intensely enhancing mass within the left periorbital/orbital soft tissues with posterior extension to the left cavernous sinus and the left cerebellopontine angle $(B)$. In addition, focal enhancement is seen within the left internal auditory canal $(D) . C$, Tortuous vessels are noted adjacent to the left internal carotid artery terminus.
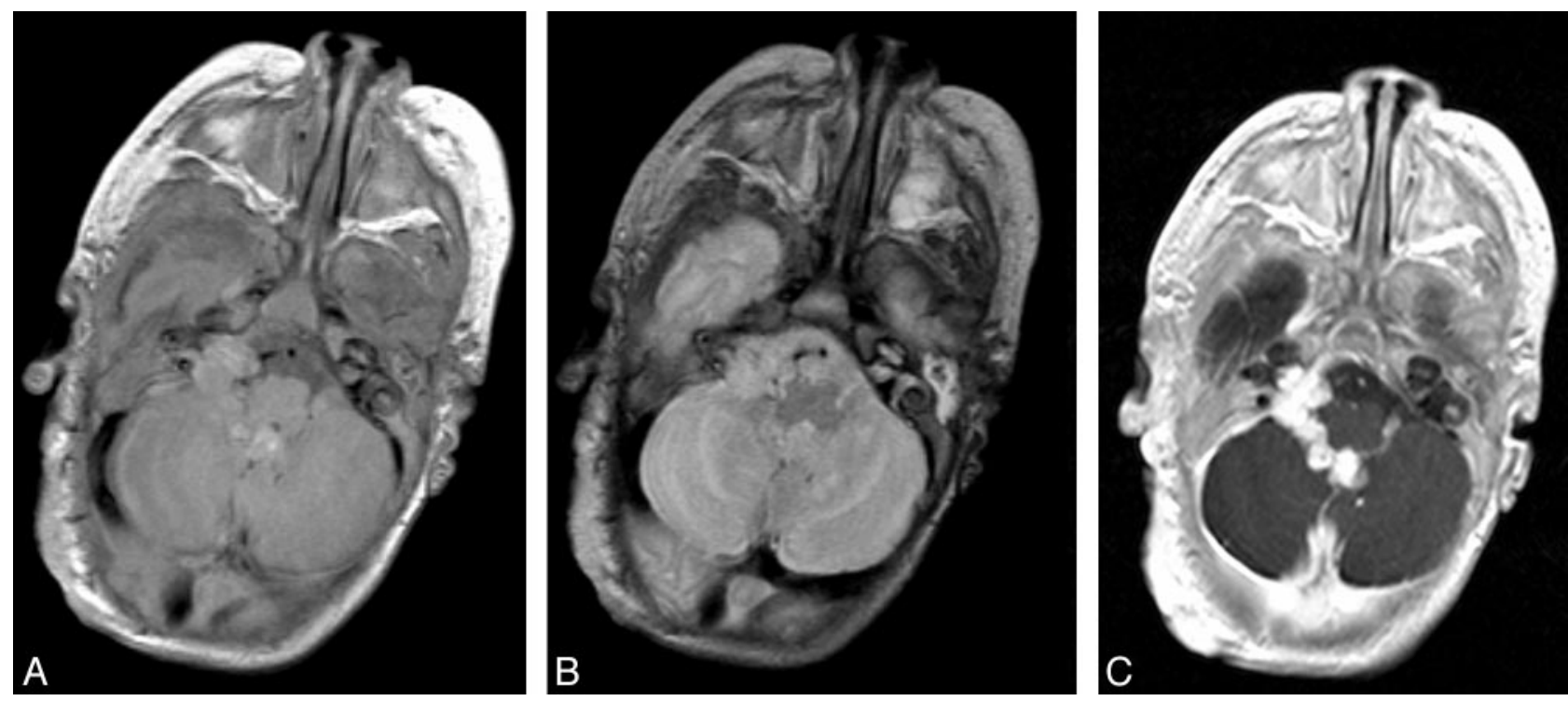

Fig 5. Infantile hemangioma in the right cerebellopontine angle cistern (patient 6$)$. Axial T1 precontrast $(A)$, axial T2 $(B)$, and axial T1 postcontrast $(C)$ images demonstrate a lobulated well-circumscribed avidly enhancing hemangioma in the fourth ventricle with extension through the foramen of Luschka to the right cerebellopontine angle cistern. 

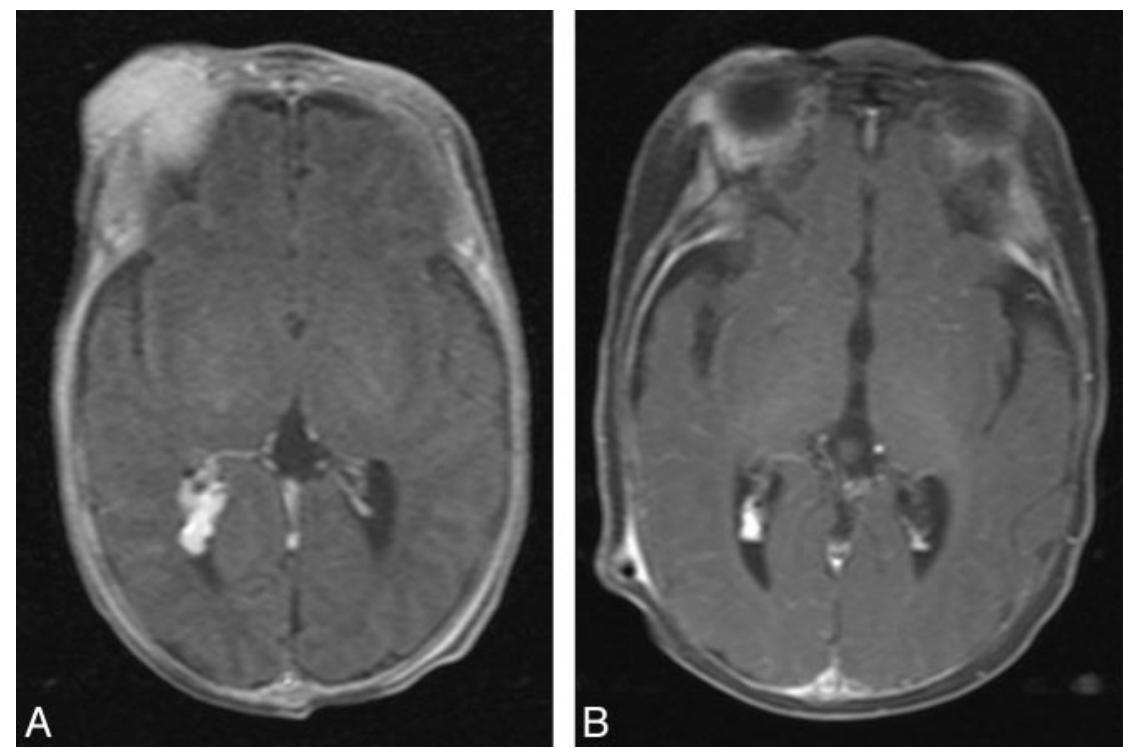

Fig 6. Concomitant regression of extracranial (right orbital/periorbital soft tissues) and intracranial (right lateral ventricle) components of infantile hemangioma (patient 9). $A$, Postcontrast axial T1-weighted pretreatment image. B, Postcontrast axial T1-weighted image after 3 months of corticosteroid treatment.

istered by the primary physician as a first-line treatment, was later switched to corticosteroid after referral to our center.

Of the 13 patients treated with corticosteroid, the lesions in 8 patients evidenced accelerated regression and 2 stabilized. Two patients were lost to follow-up, and another patient had not yet undergone follow-up imaging. Figure 6 demonstrates regression of both the extracranial (right orbital/periorbital soft tissues) and intracranial (right lateral ventricle) components of infantile hemangioma. Figure 7 illustrates regression of the prespinal and intraspinal extrathecal extension of hemangioma in response to corticosteroid treatment. In 2 patients, blepharoptosis improved with corticosteroid treatment, and in another, ocular proptosis decreased. The patient with tracheal compression by hemangioma responded to corticosteroid as well. In 1 patient with spinal hemangioma, there was improved lower extremity sensory and motor function after surgical debulking. One patient died due to intracranial hemorrhage from a concomitant arteriovenous malformation (AVM) located in the left cerebellar hemisphere, adjacent to the infantile hemangioma in the left cerebellopontine angle.

\section{Discussion}

The binary classification of vascular anomalies as tumors or malformations has been adopted by most disciplines involved in this field, ${ }^{2}$ and since 1996, the ISSVA has officially accepted the term "infantile hemangioma." Nevertheless, there has not generally been similar acceptance in the clinical neuroscience specialties. Older terminology is still used, including "strawberry mark," "capillary hemangioma," "cavernous hemangioma," "angioma," "port-wine stain," "nevus flammeus," "benign hemangioendothelioma," "Masson's vegetant intravascular hemangioendothelioma,"21 "Cobb syndrome,"22 and "angioblastomatosis," which conflates infantile hemangiomas with a host of other lesions, all of which have distinct natural histories, imaging characteristics, and treatment protocols. Of the lesions listed above, only those previously known as capillary, capillary/cavernous, and strawberry hemangiomas are histologically identical to what is now categorized as "infantile hemangiomas." 23

The differential diagnosis of vascular lesions involving the neuraxis in infancy includes hemangioma, hemangioblastoma, vascular malformation, and other neonatal tumors, such as soft-tissue sarcoma. The presence of radiologically indistinguishable extracranial tumor and concordant changes in size of the extra-CNS and CNS lesions should suggest neuraxial hemangioma. ${ }^{5}$ In contrast, hemangioblastoma often has a cyst with an associated enhancing nodule. Unlike hemangioma, soft-tissue sarcoma is often heterogeneous and exhibits invasive characteristics. Cerebral cavernous malformation (CCM) appears as a reticulated mass with heterogeneous signal intensity and typically a rim of decreased signal intensity on T2-weighted images; CCM has a propensity for hemorrhage, both subclinical microbleeding and large spaceoccupying hematomas. AVMs demonstrate prominent flow voids, lack of soft-tissue mass, growth proportionate with the child's age, and early venous shunting. Other neonatal tumors do not regress in the manner of infantile hemangioma.

Fifteen cases of true intracranial infantile hemangiomas have been reported previously. They have been described as having a predilection for the posterior fossa, especially the fourth ventricle and the cerebellopontine angle cistern. ${ }^{3-8}$ Other reported sites include the pineal region, the hypothalamus, the hippocampus, and along the cerebellar convexity. ${ }^{4,5}$ There is 1 recent case report of diffuse neonatal hemangiomatosis that involved the brain and cervical spinal cord. ${ }^{9}$ Other associated findings reported included cerebellar hypoplasia and atrophy, persistent trigeminal artery, periventricular gray matter heterotopia, cerebral AVM in 1 patient, Dandy-Walker complex, and multifocal hepatic hemangiomas. ${ }^{3-5,8,24} \mathrm{Re}-$ ported examples of intraspinal hemangiomas are even rarer, numbering 5 .

In this series, we documented cutaneous hemangiomas in 15 patients with involvement of the neuraxis, representing approximately $1 \%$ of all hemangiomas referred to our center. 

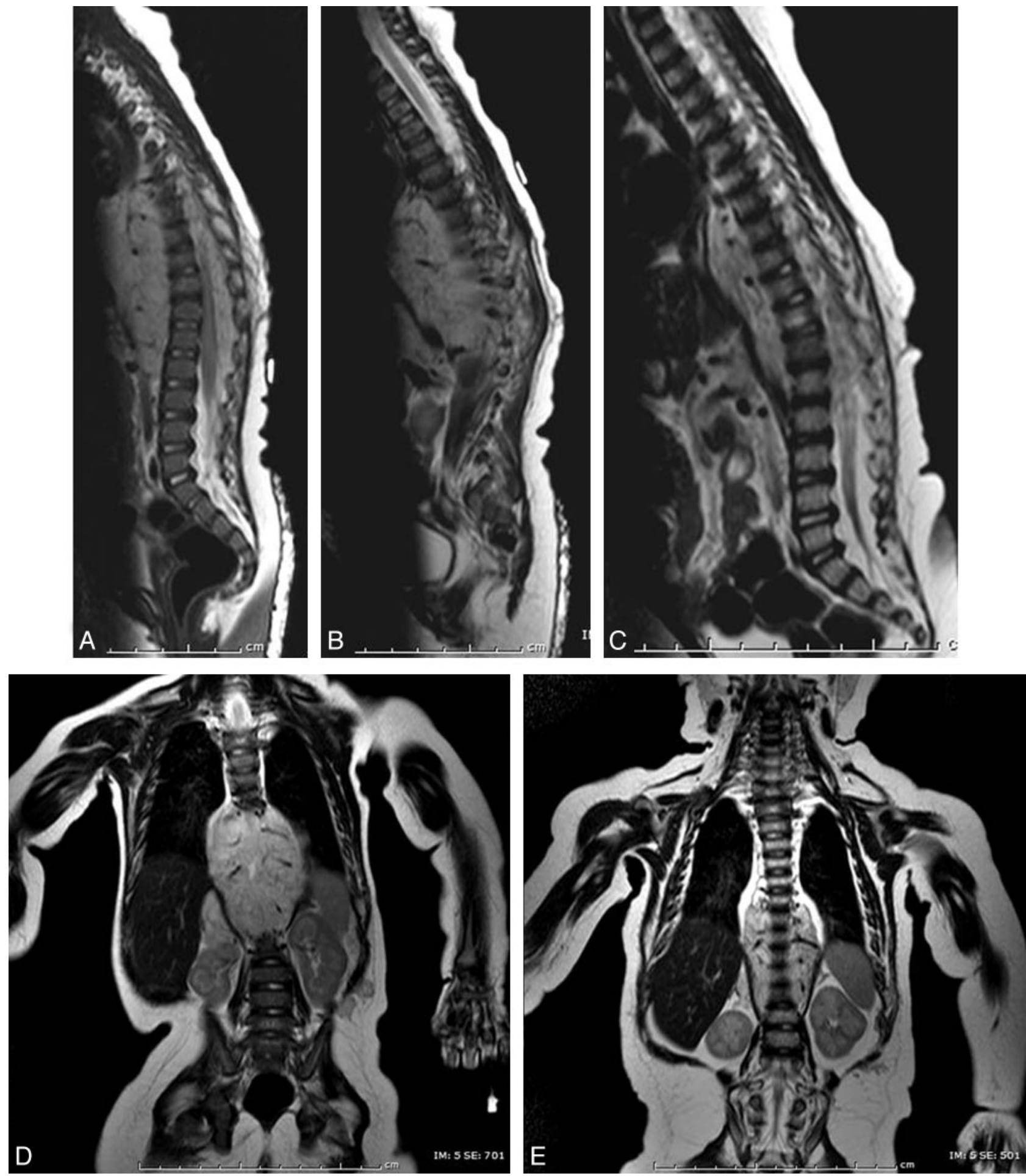

Fig 7. Regression of the prespinal and extradural extension of hemangioma in response to corticosteroid treatment (patient 14). Paramedian sagittal and coronal T2-weighted pretreatment images $(A, B$, and $D)$ and midine sagittal and coronal T2-weighted images after 3 months of corticosteroid treatment $(C$ and $E)$.

Because uncomplicated hemangiomas are rarely sent to us for consultation, the true prevalence of neuraxis involvement by infantile hemangioma is very likely significantly lower.

None of the neuraxial hemangiomas in our cohort demonstrated parenchymal invasion of the CNS. This contrasts with a recent report of diffuse neonatal hemangiomatosis with parenchymal involvement. ${ }^{9}$ In our cases, the hemangioma insinuated along extra-axial cisternal, intraventricu- lar, and subarachnoid spaces. Hemangioma has been described to have an affinity for the cerebellopontine angle cistern and the fourth ventricle, ${ }^{4-8}$ and we observed this distribution in several of our patients. In 1 of our patients, intracranial hemangioma extended posteriorly from the left orbit into the cavernous sinus and to the left cerebellopontine angle cistern. This pattern has also been described previously. ${ }^{6}$ The internal auditory canal, the cav- 
ernous sinus, Meckel cave, and other adjacent structures were involved in our patients as well. All of our patients with spinal hemangioma showed extradural tumor. Of the 2 patients with tethered cord, 1 patient had an associated intramedullary angiolipofibroma and extradural hemangioma.

Several associated findings were seen in our cohort of patients with CNS-involved hemangioma (on-line Table), including arterial anomalies, ipsilateral cerebellar hypoplasia, Dandy-Walker malformation, Moyamoya-like proliferation of the ICA terminus, and other manifestations of the PHACES association. Recent studies suggest that $\geq 2 \%$ of children with facial hemangiomas and $20 \%$ of children with regional facial hemangiomas may have PHACES association (Online Mendelian Inheritance in Man, 606519). ${ }^{25}$

Hydrocephalus and dural sinus thrombosis, not previously described in the context of neuraxis hemangiomas, were seen in our cohort in 3 patients and 1 patient, respectively. It is unclear whether these findings represent sequelae of intracranial hemangioma or merely associations. There were 2 cases with internal hemangioma elsewhere (tracheal and gastrointestinal), 1 case with associated lipoma, and 1 with angiolipofibroma. Hemolysis, elevated liver enzyme levels, and low platelet levels and premature rupture of membranes were diagnosed in 2 mothers whose infants subsequently developed neuraxial hemangioma. One patient with intracranial hemangioma of the basal cisterns and the pineal region also had a left cerebellar hemisphere AVM. The AVM was embolized at ages 3 and 4 at another institution, and the patient died of complications related to intracranial hemorrhage 2 years later. ${ }^{4}$ The presence of both AVM and hemangioma is likely coincidental.

Given the risk of associated CNS and cerebrovascular abnormalities, further clinical and imaging investigation has been recommended for hemangioma in a regional facial distribution. ${ }^{3}$ We would recommend contrast-enhanced brain MR imaging and intracranial MR angiography in this setting. Imaging has also been recommended whenever a cutaneous hemangioma lies over the lumbosacral spine, in perineal hemangiomas with associated urogenital or anal abnormalities, when more than 4 cutaneous hemangiomas are present, and for hemangioma with atypical presentation such as thrombocytopenia. $^{26}$ The reticular variant of hemangioma has been associated with underlying ventral-caudal structural anomalies. ${ }^{11}$ As noted, reticular hemangioma was present in 2 of our patients with intraspinal extension. Thus, the presence of reticular spinal hemangioma also warrants imaging follow-up with contrast-enhanced spinal MR imaging.

There are several limitations to this study. First, it is retrospective rather than prospective. However, given the low incidence of neuraxial involvement by infantile hemangioma, a prospective study would require screening many thousands of patients with the tumor, most of whom would not be referred to tertiary care. Second, given the wide catchment area of the Vascular Anomalies Center, many of our patients were treated elsewhere, and we do not have complete imaging and clinical history follow-up available for each patient. Similarly, the time course and specific medication regimen were not available in some cases due to incomplete medical records. The small patient sample size of 15 is likewise a limitation but is a manifes- tation of the low incidence of CNS involvement, as mentioned above.

\section{Conclusions}

In summary, the ISSVA classification of vascular anomalies has been widely accepted by most specialties involved in treating infantile hemangiomas. Proper clinical management hinges on careful identification of infantile hemangioma, with its unique natural history, response to appropriate therapy, and the pattern of CNS involvement described here. Unfortunately, numerous vascular lesions involving the CNS have been and continue to be labeled "hemangioma." Misclassification as either vascular anomalies or aggressive vascular tumors may lead to improper management.

As shown in this study, the prevalence of neuraxial involvement by hemangioma of infancy is extremely low. When it does occur, there is a predilection for neuraxial hemangiomas to involve the basal cisterns, the ventricular system, and the extradural spine. Surveillance imaging for potential rare associations, such as hydrocephalus or sinus thrombosis, is helpful for optimal patient care.

\section{Acknowledgments}

We are grateful for the assistance of Mary Beth Sylvia, Erin Ryan, and Kimberly Chalache of the Vascular Anomalies Center at Children's Hospital Boston. We also thank the many clinicians who referred their patients to our institution.

\section{References}

1. Mulliken JB, Glowacki J. Hemangiomas and vascular malformations in infants and children: a classification based on endothelial characteristics. Plast Reconstr Surg 1982;69:412-22

2. Enjolras O, Mulliken JB. Vascular tumors and vascular malformations (new issues). Adv Dermatol 1997;13:375-423

3. Oza VS, Wang E, Berenstein A, et al. PHACES association: a neuroradiologic review of 17 patients. AJNR Am J Neuroradiol 2008;29:807-13

4. Song JK, Niimi Y, Kupersmith MJ, et al. Postnatal growth and development of a cerebral arteriovenous malformation on serial magnetic resonance imaging in a child with hemangiomatosis: case report. J Neurosurg 2007;106(5 suppl):384-87

5. Tortori-Donati P, Fondelli MP, Rossi A, et al. Intracranial contrast-enhancing masses in infants with capillary haemangioma of the head and neck: intracranial capillary haemangioma? Neuroradiology 1999;41:369-75

6. Ersoy S, Mancini AJ. Hemifacial infantile hemangioma with intracranial extension: a rare entity. Pediatr Dermatol 2005;22:309-13

7. Karikari IO, Selznick LA, Cummings TJ, et al. Capillary hemangioma of the fourth ventricle in an infant: case report and review of the literature. J Neurosurg 2006;104:188-91

8. Poindexter G, Metry DW, Barkovich AJ, et al. PHACE syndrome with intracerebral hemangiomas, heterotopia, and endocrine dysfunction. Pediatr Neurol 2007;36:402-06

9. Balaci E, Sumner TE, Auringer ST, et al. Diffuse neonatal hemangiomatosis with extensive involvement of the brain and cervical spinal cord. Pediatr Radiol 1999;29:441-43

10. Ganapathy S, Kleiner LI, Mirkin LD, et al. Intradural capillary hemangioma of the cauda equina. Pediatr Radiol 2008;38:1235-8. Epub 2008 Jul 29

11. Mulliken JB, Marler JJ, Burrows PE, et al. Reticular infantile hemangioma of the limb can be associated with ventral-caudal anomalies, refractory ulceration, and cardiac overload. Pediatr Dermatol 2007;24:356-62

12. Karikari IO, Selznick LA, Cummings TJ, et al. Spinal capillary hemangioma in infants: report of two cases and review of the literature. Pediatr Neurosurg 2007;43:125-29

13. Finn MC, Glowacki J, Mulliken JB. Congenital vascular lesions: clinical application of a new classification. J Pediatr Surg 1983;18:894-900

14. Burrows PE, Laor T, Paltiel H, et al. Diagnostic imaging in the evaluation of vascular birthmarks. Dermatol Clin 1998;16:455-88

15. Willing SJ, Faye-Petersen O, Aronin P, et al. Radiologic-pathologic correlation capillary hemangioma of the meninges. AJNR Am J Neuroradiol 1993; 14:529-36 
16. Dillon WP, Som PM, Rosenau W. Hemangioma of the nasal vault: MR and CT features. Radiology 1991;180:761-65

17. Dubois J, Patriquin HB, Garel L, et al. Soft-tissue hemangiomas in infants and children: diagnosis using Doppler sonography. AJR Am J Roentgenol 1998;171: 247-52

18. Paltiel HJ, Burrows PE, Kozakewich HP, et al. Soft-tissue vascular anomalies: utility of US for diagnosis. Radiology 2000;214:747-54

19. Burrows PE, Mulliken JB, Fellows KE, et al. Childhood hemangiomas and vascular malformations: angiographic differentiation. AJR Am J Roentgenol 1983;141:483-88

20. North PE, Waner M, Mizeracki A, et al. GLUT1: a newly discovered immunohistochemical marker for juvenile hemangiomas. Hum Pathol 2000;31:11-22

21. Nagib MG, Sung JH, Seljeskog EL. Neurocutaneous Masson's vegetant intravascular hemangioendothelioma. Neurosurgery 1982;11:800-03

22. Maramattom BV, Cohen-Gadol AA, Wijdicks EF, et al. Segmental cutaneous hemangioma and spinal arteriovenous malformation (Cobb syndrome): case report and historical perspective. J Neurosurg Spine 2005;3:249-52

23. Fordham LA, Chung CJ, Donnelly LF. Imaging of congenital vascular and lymphatic anomalies of the head and neck. Neuroimaging Clin N Am 2000;10: 117-36, viii

24. Bar-Sever Z, Horev G, Lubin E, et al. A rare coexistence of a multicentric hepatic hemangioendothelioma with a large brain hemangioma in a preterm infant. Pediatr Radiol 1994;24:141-42

25. Metry DW, Haggstrom AN, Drolet BA, et al. A prospective study of PHACE syndrome in infantile hemangiomas: demographic features, clinical findings, and complications. Am J Med Genet A 2006;140:975-86

26. Frieden IJ, Haggstrom AN, Drolet BA, et al. Infantile hemangiomas: curren knowledge, future directions-Proceedings of a research workshop on infantile hemangiomas, April 7-9, 2005, Bethesda, Maryland, USA. Pediatr Dermatol 2005;22:383-406 